Pacific

Journal of

Mathematics

\title{
A PROOF OF THE DDVV CONJECTURE AND ITS EQUALITY CASE
}

GE JiANQUAN AND TANG ZiZHOU 


\title{
A PROOF OF THE DDVV CONJECTURE AND ITS EQUALITY CASE
}

\author{
GE JIANQUAN AND TANG ZiZHOU
}

\begin{abstract}
We give a proof of the DDVV conjecture, which is a pointwise inequality involving the scalar curvature, the normal scalar curvature and the mean curvature on a submanifold of a real space form. We also solve the problem of its equality case.
\end{abstract}

\section{Introduction}

Let $f: M^{n} \rightarrow N^{n+m}(c)$ be an isometric immersion of an $n$-dimensional submanifold $M$ into the $(n+m)$-dimensional real space form $N^{n+m}(c)$ of constant sectional curvature $c$. The normalized scalar curvature $\rho$ and normal scalar curvature $\rho^{\perp}$ are defined by [DDVV 1999]

$$
\begin{aligned}
\rho & =\frac{2}{n(n-1)} \sum_{1=i<j}^{n} R\left(e_{i}, e_{j}, e_{j}, e_{i}\right), \\
\rho^{\perp} & =\frac{2}{n(n-1)}\left(\sum_{1=i<j}^{n} \sum_{1=r<s}^{m}\left\langle R^{\perp}\left(e_{i}, e_{j}\right) \xi_{r}, \xi_{s}\right\rangle^{2}\right)^{1 / 2},
\end{aligned}
$$

where $\left\{e_{1}, \ldots, e_{n}\right\}$ is an orthonormal basis of the tangent space, and $R$ is the curvature tensor of the tangent bundle. Similarly, $\left\{\xi_{1}, \ldots, \xi_{m}\right\}$ is an orthonormal basis of normal space, and $R^{\perp}$ is the curvature tensor of the normal bundle.

Let $h$ be the second fundamental form and let $H=(1 / n) \operatorname{Tr} h$ be the mean curvature vector field. The DDVV conjecture, of De Smet, Dillen, Verstraelen, and Vrancken [1999], says that there's a pointwise inequality among $\rho, \rho^{\perp}$, and $|H|^{2}$ given by

$$
\rho+\rho^{\perp} \leq|H|^{2}+c .
$$

Since this is a pointwise inequality, one can see using the Gauss and Ricci identities that it's equivalent to the following algebraic inequality (see [Dillen et al. 2007]):

MSC2000: 53C42, 15A45.

Keywords: normal scalar curvature, mean curvature, commutator.

The project is partially supported by the NSFC (numbers 10531090 and 10229101) and the Chang Jiang Scholars Program.

Tang Zizhou is the corresponding author. 
Conjecture A. Let $B_{1}, \ldots, B_{m}$ be $(n \times n)$ real symmetric matrices. Then

$$
\sum_{r, s=1}^{m}\left\|\left[B_{r}, B_{s}\right]\right\|^{2} \leq\left(\sum_{r=1}^{m}\left\|B_{r}\right\|^{2}\right)^{2},
$$

where $\|\cdot\|^{2}$ denotes the sum of the squares of the entries of the matrix and $[A, B]=$ $A B-B A$ is the commutator of the matrices $A$ and $B$.

The main purpose of this paper is to prove Conjecture A and also to give the equality condition:

Theorem 1.1. Let $B_{1}, \ldots, B_{m}$ be $(n \times n)$ real symmetric matrices. Then

$$
\sum_{r, s=1}^{m}\left\|\left[B_{r}, B_{s}\right]\right\|^{2} \leq\left(\sum_{r=1}^{m}\left\|B_{r}\right\|^{2}\right)^{2},
$$

where the equality holds if and only if under some rotation ${ }^{1}$ all $B_{r}$ 's are zero except two matrices which can be written as $P H_{1} P^{t}$ and $P H_{2} P^{t}$, where $P$ is an $(n \times n)$ orthogonal matrix, and

$$
H_{1}=\operatorname{diag}(\mu,-\mu, 0, \ldots), \quad H_{2}=\operatorname{diag}\left(\left(\begin{array}{cc}
0 & \mu \\
\mu & 0
\end{array}\right), 0, \ldots\right) .
$$

Therefore, we can solve the DDVV conjecture also with its equality conditions in terms of shape operators:

Corollary 1.2. Let $f: M^{n} \rightarrow N^{n+m}(c)$ be an isometric immersion. Then

$$
\rho+\rho^{\perp} \leq|H|^{2}+c,
$$

where the equality holds at some point $p \in M$ if and only if there exist an orthonormal basis $\left\{e_{1}, \ldots, e_{n}\right\}$ of $T_{p} M$ and an orthonormal basis $\left\{\xi_{1}, \ldots, \xi_{m}\right\}$ of $T_{p}^{\perp} M$, such that

$$
A_{\xi_{1}}=\operatorname{diag}\left(\lambda_{1}+\mu, \lambda_{1}-\mu, \lambda_{1}, \ldots, \lambda_{1}\right), \quad A_{\xi_{2}}=\operatorname{diag}\left(\left(\begin{array}{cc}
\lambda_{2} & \mu \\
\mu & \lambda_{2}
\end{array}\right), \lambda_{2}, \ldots, \lambda_{2}\right),
$$

and all other shape operators $A_{\xi_{r}}=\lambda_{r} I_{n}$, where $\mu, \lambda_{1}, \ldots, \lambda_{m}$ are real numbers.

Remark. By the same method, one can see that Conjecture A also holds (though not optimally) for antisymmetric matrices. However, the following example shows that Conjecture A fails, as conjectured in [Lu 2007c], when the set $\left\{B_{1}, \ldots, B_{m}\right\}$ contains both symmetric and antisymmetric matrices.

Example. The conclusion of Conjecture A fails when

$$
B_{1}=\left(\begin{array}{rr}
1 & 0 \\
0 & -1
\end{array}\right), \quad B_{2}=\left(\begin{array}{ll}
0 & 1 \\
1 & 0
\end{array}\right), \quad B_{3}=\left(\begin{array}{rr}
0 & 1 \\
-1 & 0
\end{array}\right) .
$$

\footnotetext{
${ }^{1}$ An orthogonal $m \times m$ matrix $R=\left(R_{r s}\right)$ acts as a rotation on $\left(B_{1}, \ldots, B_{m}\right)$ by $R\left(B_{r}\right)=$ $\sum_{s=1}^{m} R_{s r} B_{s}$.
} 
We point out that the inequality and its equality condition of Theorem 1.1 (respectively Corollary 1.2) for $m=2$ were given in [Chern 1968] (respectively [DDVV 1999]). There are also some studies about classifying submanifolds that satisfy the equality everywhere, for example [Choi and Lu 2008; DDVV 1999; Dajczer and Florit 2001; Dillen et al. 2007]. The first nontrivial case, $n=3$, of the DDVV conjecture was proved in [Choi and Lu 2008]. For general $n$ and $m$, a weaker version was proved in [Dillen et al. 2007]. After we solved the conjecture and its equality case, we found very recently that Zhiqin Lu had proved the inequality without the equality case (the inequality's key step is contained in [Lu 2007a; Lu 2007c]), as seen on his homepage [Lu 2007b]. Since we use a quite different method and work out the equality condition in addition to the inequality, we'd like it to be part of the literature.

\section{Notations and preparatory lemmas}

Throughout this paper, we denote by $\mathrm{M}(m, n)$ the space of $m \times n$ real matrices, $\mathrm{M}(n)$ the space of $n \times n$ real matrices, and $\mathrm{SM}(n)$ the subspace of symmetric matrices in $\mathrm{M}(n)$, which has dimension $N:=n(n+1) / 2$.

For every $(i, j)$ with $1 \leq i \leq j \leq n$, let

$$
\hat{E}_{i j}:= \begin{cases}E_{i i} & \text { if } i=j, \\ \left(E_{i j}+E_{j i}\right) / \sqrt{2} & \text { if } i<j,\end{cases}
$$

where $E_{i j} \in \mathrm{M}(n)$ is the matrix with 1 in position $(i, j)$ and 0 elsewhere. Clearly $\left\{\hat{E}_{i j}\right\}_{i \leq j}$ is an orthonormal basis of $\operatorname{SM}(n)$. Let us put an ordering on the index set $S:=\{(i, j) \mid 1 \leq i \leq j \leq n\}$ by saying

$$
(i, j)<(k, l) \text { if and only if } i<k \text { or } i=k \text { and } j<l \text {. }
$$

We use this ordering to index elements of $S$ with a single (Greek) index in the range $\{1, \ldots, N\}$.

For $\alpha=(i, j)<(k, l)=\beta$ in $S$, direct calculations imply

$$
\left\|\left[\hat{E}_{\alpha}, \hat{E}_{\beta}\right]\right\|^{2}= \begin{cases}1 & \text { if } i=j=k<l \text { or } i<j=k=l \\ 1 / 2 & \text { if } i<j=k<l \text { or } i=k<j<l \text { or } i<k<j=l, \\ 0 & \text { otherwise, }\end{cases}
$$

and for any $\alpha, \beta \in S$,

$$
\sum_{\gamma \in S}\left\langle\left[\hat{E}_{\alpha}, \hat{E}_{\gamma}\right],\left[\hat{E}_{\beta}, \hat{E}_{\gamma}\right]\right\rangle=n \delta_{\alpha \beta}-\delta_{\alpha} \delta_{\beta},
$$

where $\delta_{\alpha \beta}=\delta_{i k} \delta_{j l}, \delta_{\alpha}=\delta_{i j}, \delta_{\beta}=\delta_{k l}$, and $\langle\cdot, \cdot\rangle$ is the standard inner product of $\mathrm{M}(n)$. 
Let $\left\{\hat{Q}_{\alpha}\right\}_{\alpha \in S}$ be any orthonormal basis of $\operatorname{SM}(n)$. Then there exists a unique orthogonal matrix $Q \in O(N)$ such that $\left(\hat{Q}_{1}, \ldots, \hat{Q}_{N}\right)=\left(\hat{E}_{1}, \ldots, \hat{E}_{N}\right) Q$, that is, $\hat{Q}_{\alpha}=\sum_{\beta} q_{\beta \alpha} \hat{E}_{\beta}$ for $Q=\left(q_{\alpha \beta}\right)_{N \times N}$, and if $\hat{Q}_{\alpha}=\left(\hat{q}_{i j}^{\alpha}\right)_{n \times n}$,

$$
\hat{q}_{i j}^{\alpha}=\hat{q}_{j i}^{\alpha}= \begin{cases}q_{\beta \alpha} & \text { if } \beta=(i, j) \text { and } i=j, \\ q_{\beta \alpha} / \sqrt{2} & \text { if } \beta=(i, j) \text { and } i<j .\end{cases}
$$

Let $\lambda_{1}, \ldots, \lambda_{n}$ be $n$ real numbers satisfying $\sum_{i} \lambda_{i}^{2}=1$ and $\lambda_{1} \geq \cdots \geq \lambda_{n}$. Define $I_{1}:=\left\{j \mid \lambda_{1}-\lambda_{j}>1\right\}, I_{2}:=\left\{i \mid \lambda_{i}-\lambda_{n}>1\right\}$, and $I:=\left\{(i, j) \mid \lambda_{i}-\lambda_{j}>1\right\}$. Let $n_{0}$ be the number of elements of $I$. Then $\left(\{1\} \times I_{1}\right) \cup\left(I_{2} \times\{n\}\right) \subset I \subset S$.

Lemma 2.1. Either $I=\{1\} \times I_{1}$ or $I=I_{2} \times\{n\}$.

Proof. If $n_{0}=0$, the three sets are all empty. If $n_{0}=1$, the single element must be $(1, n)$, and the three sets are equal. Now let $(1, n)$ and $\left(i_{1}, j_{1}\right)$ be two different elements of $I$, that is, $\lambda_{1}-\lambda_{n} \geq \lambda_{i_{1}}-\lambda_{j_{1}}>1$ and $(1, n) \neq\left(i_{1}, j_{1}\right)$. We assert that either $i_{1}=1$ and $j_{1} \neq n$ or $i_{1} \neq 1$ and $j_{1}=n$, which shows exactly that $I=\{1\} \times I_{1} \bigcup I_{2} \times\{n\}$. Otherwise, $1, i_{1}, j_{1}$, and $n$ will be four different elements in $\{1, \ldots, n\}$, and thus

$$
1 \geq \lambda_{1}^{2}+\lambda_{i_{1}}^{2}+\lambda_{j_{1}}^{2}+\lambda_{n}^{2} \geq \frac{1}{2}\left(\lambda_{1}-\lambda_{n}\right)^{2}+\frac{1}{2}\left(\lambda_{i_{1}}-\lambda_{j_{1}}\right)^{2}>1
$$

is a contradiction. Without loss of generality, we can assume $\left(i_{1}, j_{1}\right) \in\{1\} \times I_{1}$. Then it'll be seen that $I_{2} \times\{n\}=\{(1, n)\}$ and thus $I=\{1\} \times I_{1}$, which completes the proof. Otherwise, if there's another element, say $\left(i_{2}, n\right)$, in $I_{2} \times\{n\}$, then $i_{1}=1$, $j_{1}, i_{2}$, and $n$ are four different elements in $\{1, \ldots, n\}$, and we come to the same contradiction as above.

Lemma 2.2. We have $\sum_{(i, j) \in I}\left[\left(\lambda_{i}-\lambda_{j}\right)^{2}-1\right] \leq 1$, where the equality holds in the case when $I=\{1\} \times I_{1}$ if and only if $1 \leq n_{0}<n$ and $\lambda_{1}=\sqrt{n_{0} /\left(n_{0}+1\right)}$, $\lambda_{n-n_{0}+1}=\cdots=\lambda_{n}=-1 / \sqrt{n_{0}^{2}+n_{0}}$ and all other $\lambda_{k}=0$.

Proof. Without loss of generality, we can assume that $I=\{1\} \times I_{1}$ by Lemma 2.1. Then

$$
\begin{aligned}
\sum_{(i, j) \in I}\left[\left(\lambda_{i}-\lambda_{j}\right)^{2}-1\right] & =\sum_{j \in I_{1}}\left(\lambda_{1}^{2}+\lambda_{j}^{2}-2 \lambda_{1} \lambda_{j}\right)-n_{0} \\
& =n_{0} \lambda_{1}^{2}+\sum_{j \in I_{1}} \lambda_{j}^{2}-2 \lambda_{1} \sum_{j \in I_{1}} \lambda_{j}-n_{0} \\
& \leq\left(n_{0}+1\right) \lambda_{1}^{2}+\sum_{j \in I_{1}} \lambda_{j}^{2}+\left(\sum_{j \in I_{1}} \lambda_{j}\right)^{2}-n_{0} \\
& \leq\left(n_{0}+1\right)\left(\lambda_{1}^{2}+\sum_{j \in I_{1}} \lambda_{j}^{2}\right)-n_{0} \\
& \leq\left(n_{0}+1\right) \sum_{i} \lambda_{i}^{2}-n_{0}=1,
\end{aligned}
$$

where the equality condition is easily seen from the proof.

Lemma 2.3. We have $\sum_{\beta \in J_{\alpha}}\left(\left\|\left[\hat{Q}_{\alpha}, \hat{Q}_{\beta}\right]\right\|^{2}-1\right) \leq 1$ for any $Q \in O(N), \alpha \in S$ and $J_{\alpha} \subset S$. 
Proof. For $\alpha \in S$, we can assume without loss of generality $\hat{Q}_{\alpha}=\operatorname{diag}\left(\lambda_{1}, \ldots, \lambda_{n}\right)$ with $\sum_{i} \lambda_{i}^{2}=1$ and $\lambda_{1} \geq \cdots \geq \lambda_{n}$. Then by Lemma 2.2 ,

$$
\begin{aligned}
\sum_{\beta \in J_{\alpha}}\left(\left\|\left[\hat{Q}_{\alpha}, \hat{Q}_{\beta}\right]\right\|^{2}-1\right) & =\sum_{\beta \in J_{\alpha}} \sum_{i, j=1}^{n}\left(\left(\lambda_{i}-\lambda_{j}\right)^{2}-1\right)\left(\hat{q}_{i j}^{\beta}\right)^{2} \\
& =\sum_{\beta \in J_{\alpha}} \sum_{(i, j)=\gamma \in S}\left(\left(\lambda_{i}-\lambda_{j}\right)^{2}-1\right) q_{\gamma \beta}^{2} \\
& \leq \sum_{(i, j)=\gamma \in I}\left(\left(\lambda_{i}-\lambda_{j}\right)^{2}-1\right) \sum_{\beta \in J_{\alpha}} q_{\gamma \beta}^{2} \\
& \leq \sum_{(i, j) \in I}\left(\left(\lambda_{i}-\lambda_{j}\right)^{2}-1\right) \leq 1 .
\end{aligned}
$$

Lemma 2.4. We have $\sum_{\beta \in S}\left\|\left[\hat{Q}_{\alpha}, \hat{Q}_{\beta}\right]\right\|^{2} \leq n$ for any $Q \in O(N)$ and $\alpha \in S$. Proof. It follows from Equation (2-3) that

$$
\begin{aligned}
\sum_{\beta \in S}\left\|\left[\hat{Q}_{\alpha}, \hat{Q}_{\beta}\right]\right\|^{2} & =\sum_{\beta \gamma \tau \xi \eta} q_{\gamma \alpha} q_{\xi \alpha} q_{\tau \beta} q_{\eta \beta}\left\langle\left[\hat{E}_{\gamma}, \hat{E}_{\tau}\right],\left[\hat{E}_{\xi}, \hat{E}_{\eta}\right]\right\rangle \\
& =\sum_{\gamma \xi} q_{\gamma \alpha} q_{\xi \alpha} \sum_{\tau}\left\langle\left[\hat{E}_{\gamma}, \hat{E}_{\tau}\right],\left[\hat{E}_{\xi}, \hat{E}_{\tau}\right]\right\rangle \\
& =\sum_{\gamma \xi} q_{\gamma \alpha} q_{\xi \alpha}\left(n \delta_{\gamma \xi}-\delta_{\gamma} \delta_{\xi}\right)=n \sum_{\gamma} q_{\gamma \alpha}^{2}-\left(\sum_{i} \hat{q}_{i i}^{\alpha}\right)^{2} \leq n
\end{aligned}
$$

Now let $\varphi: \mathrm{M}(m, n) \rightarrow \mathrm{M}\left(C_{m}^{2}, C_{n}^{2}\right)$ be the map defined by $\varphi(A)_{(i, j)(k, l)}:=A\left({ }_{i j}^{k l}\right)$, where $1 \leq i<j \leq m, 1 \leq k<l \leq n$, and $A\left(\begin{array}{c}k l \\ i j\end{array}\right)=a_{i k} a_{j l}-a_{i l} a_{j k}$ is the discriminant of the $2 \times 2$ submatrix of $A$ that is the intersection of rows $i$ and $j$ with columns $k$ and $l$, arranged with the same order as in (2-1). We have easily $\varphi\left(I_{n}\right)=I_{C_{n}^{2}}$, $\varphi(A)^{t}=\varphi\left(A^{t}\right)$, and the following lemma.

Lemma 2.5. The map $\varphi$ preserves the matrix product, that is, $\varphi(A B)=\varphi(A) \varphi(B)$ holds for $A \in \mathrm{M}(m, k)$ and $B \in \mathrm{M}(k, n)$.

We'll also need a result of linear algebra for proving the equality case.

Lemma 2.6. Let $A, B$ be two matrices in $\mathrm{M}(m, n)$. Then $A A^{t}=B B^{t}$ if and only if $A=B R$ for some $R \in O(n)$.

\section{Proof of the main results}

Let $B_{1}, \ldots, B_{m}$ be any real symmetric $n \times n$ matrices. Their coefficients in the standard basis $\left\{\hat{E}_{\alpha}\right\}_{\alpha \in S}$ of $\mathrm{SM}(n)$ are determined by a matrix $B \in \mathrm{M}(N, m)$ as $\left(B_{1}, \ldots, B_{m}\right)=\left(\hat{E}_{1}, \ldots, \hat{E}_{N}\right) B$. Taking the same ordering as in (2-1) for $1 \leq r<$ 
$s \leq m$ and $1 \leq \alpha<\beta \leq N$, we arrange $\left\{\left[B_{r}, B_{s}\right]\right\}_{r<s}$ and $\left\{\left[\hat{E}_{\alpha}, \hat{E}_{\beta}\right]\right\}_{\alpha<\beta}$ into $C_{m}^{2}$ and $C_{N}^{2}$ vectors, respectively. We first observe that

$$
\left(\left[B_{1}, B_{2}\right], \ldots,\left[B_{m-1}, B_{m}\right]\right)=\left(\left[\hat{E}_{1}, \hat{E}_{2}\right], \ldots,\left[\hat{E}_{N-1}, \hat{E}_{N}\right]\right) \cdot \varphi(B) .
$$

Define a matrix $C(E)$ in $\mathrm{M}\left(C_{N}^{2}\right)$ by $C(E)_{(\alpha, \beta)(\gamma, \tau)}:=\left\langle\left[\hat{E}_{\alpha}, \hat{E}_{\beta}\right],\left[\hat{E}_{\gamma}, \hat{E}_{\tau}\right]\right\rangle$ for $1 \leq \alpha<\beta \leq N$ and $1 \leq \gamma<\tau \leq N$. We use the same notation for $\left\{B_{r}\right\}$ and $\left\{\hat{Q}_{\alpha}\right\}$, that is, we write $C(B)$ and $C(Q)$, respectively. Then it's obvious that

$$
C(B)=\varphi\left(B^{t}\right) C(E) \varphi(B) \quad \text { and } \quad C(Q)=\varphi\left(Q^{t}\right) C(E) \varphi(Q) .
$$

Since $B B^{t}$ is a semi positive definite matrix in $\operatorname{SM}(N)$, there exists an orthogonal matrix $Q \in \mathrm{SO}(N)$ such that $B B^{t}=Q \operatorname{diag}\left(x_{1}, \ldots, x_{N}\right) Q^{t}$ with $x_{\alpha} \geq 0$ for $1 \leq$ $\alpha \leq N$. Thus $\sum_{r=1}^{m}\left\|B_{r}\right\|^{2}=\|B\|^{2}=\sum_{\alpha=1}^{N} x_{\alpha}$, and hence by Lemma 2.5

$$
\begin{aligned}
\sum_{r, s=1}^{m}\left\|\left[B_{r}, B_{S}\right]\right\|^{2} & =2 \operatorname{Tr} C(B)=2 \operatorname{Tr} \varphi\left(B^{t}\right) C(E) \varphi(B)=2 \operatorname{Tr} \varphi\left(B B^{t}\right) C(E) \\
& =2 \operatorname{Tr} \varphi\left(\operatorname{diag}\left(x_{1}, \ldots, x_{N}\right)\right) C(Q)=\sum_{\alpha, \beta=1}^{N} x_{\alpha} x_{\beta}\left\|\left[\hat{Q}_{\alpha}, \hat{Q}_{\beta}\right]\right\|^{2} .
\end{aligned}
$$

Proof of Theorem 1.1. For the inequality, the arguments above show that it is equivalent to prove

$$
\sum_{\alpha, \beta=1}^{N} x_{\alpha} x_{\beta}\left\|\left[\hat{Q}_{\alpha}, \hat{Q}_{\beta}\right]\right\|^{2} \leq\left(\sum_{\alpha=1}^{N} x_{\alpha}\right)^{2} \quad \text { for any } x \in \mathbb{R}_{+}^{N} \text { and } Q \in \operatorname{SO}(N),
$$

where $\mathbb{R}_{+}^{N}:=\left\{0 \neq x=\left(x_{1}, \ldots, x_{N}\right) \in \mathbb{R}^{N} \mid x_{\alpha} \geq 0,1 \leq \alpha \leq N\right\}$ is the cone spanned by the positive axes of $\mathbb{R}^{N}$.

Let $f_{Q}(x)=F(x, Q):=\sum_{\alpha, \beta=1}^{N} x_{\alpha} x_{\beta}\left\|\left[\hat{Q}_{\alpha}, \hat{Q}_{\beta}\right]\right\|^{2}-\left(\sum_{\alpha=1}^{N} x_{\alpha}\right)^{2}$. Then $F$ is a continuous function defined on $\mathbb{R}^{N} \times \mathrm{SO}(N)$ and thus uniformly continuous on any compact subset of $\mathbb{R}^{N} \times \mathrm{SO}(N)$. Let $\triangle:=\left\{x \in \mathbb{R}_{+}^{N} \mid \sum_{\alpha} x_{\alpha}=1\right\}$ and for any sufficiently small $\varepsilon>0$, let $\triangle_{\varepsilon}:=\left\{x \in \Delta \mid x_{\alpha} \geq \varepsilon, 1 \leq \alpha \leq N\right\}$. Also let

$$
\begin{aligned}
G & :=\left\{Q \in \mathrm{SO}(N) \mid f_{Q}(x) \leq 0 \text { for all } x \in \triangle\right\}, \\
G_{\varepsilon} & :=\left\{Q \in \mathrm{SO}(N) \mid f_{Q}(x)<0 \text { for all } x \in \triangle_{\varepsilon}\right\} .
\end{aligned}
$$

We claim that $G=\lim _{\varepsilon \rightarrow 0} G_{\varepsilon}=\mathrm{SO}(N)$. Note that this implies (3-2) and thus proves the inequality. In fact we can show

$$
G_{\varepsilon}=\operatorname{SO}(N) \text { for any sufficiently small } \varepsilon>0 .
$$

To prove (3-3), we use the continuity method, in which we must prove the following three properties:

(i) $I_{N} \in G_{\varepsilon}$ (and thus $G_{\varepsilon} \neq \varnothing$ ); 
(ii) $G_{\varepsilon}$ is open in $\mathrm{SO}(N)$;

(iii) $G_{\varepsilon}$ is closed in $\mathrm{SO}(N)$.

Since $F$ is uniformly continuous on $\triangle_{\varepsilon} \times \mathrm{SO}(N)$, (ii) is obvious.

Proof of (i). For any $x \in \triangle_{\varepsilon}, f_{I_{N}}(x)=\sum_{\alpha, \beta=1}^{N} x_{\alpha} x_{\beta}\left\|\left[\hat{E}_{\alpha}, \hat{E}_{\beta}\right]\right\|^{2}-1$.

It follows from (2-2) that

$$
\begin{aligned}
f_{I_{N}}(x) & =2\left\{\sum_{i<j}\left(x_{i i} x_{i j}+x_{i j} x_{j j}\right)+\frac{1}{2} \sum_{i<j<k}\left(x_{i j} x_{j k}+x_{i j} x_{i k}+x_{i k} x_{j k}\right)\right\}-1 \\
& =2 \sum_{i<j}\left(x_{i i} x_{i j}+x_{i j} x_{j j}\right)+\sum_{i<j<k}\left(x_{i j} x_{j k}+x_{i j} x_{i k}+x_{i k} x_{j k}\right)-\left(\sum_{i \leq j}^{N} x_{i j}\right)^{2} \\
& <0
\end{aligned}
$$

which means $I_{N} \in G_{\varepsilon}$.

Proof of (iii). We only need to prove the following a priori estimate: Suppose $f_{Q}(x) \leq 0$ for every $x \in \triangle_{\varepsilon}$. Then $f_{Q}(x)<0$ for every $x \in \triangle_{\varepsilon}$.

The proof of this estimate is as follows: If there is a point $y \in \Delta_{\varepsilon}$ such that $f_{Q}(y)=0$, we can assume without loss of generality that

$$
y \in \triangle_{\varepsilon}^{\gamma}:=\left\{x \in \triangle_{\varepsilon} \mid x_{\alpha}>\varepsilon \text { for } \alpha \leq \gamma \text { and } x_{\beta}=\varepsilon \text { for } \beta>\gamma\right\}
$$

for some $1 \leq \gamma \leq N$. Then $y$ is a maximum point of $f_{Q}(x)$ in the cone spanned by $\triangle_{\varepsilon}$ and an interior maximum point in $\triangle_{\varepsilon}^{\gamma}$. Hence there exist numbers $b_{\gamma+1}, \ldots, b_{N}$ and a number $a$ such that

$$
\begin{aligned}
\left(\frac{\partial f_{Q}}{\partial x_{1}}(y), \ldots, \frac{\partial f_{Q}}{\partial x_{\gamma}}(y)\right) & =2 a(1, \ldots, 1), \\
\left(\frac{\partial f_{Q}}{\partial x_{\gamma+1}}(y), \ldots, \frac{\partial f_{Q}}{\partial x_{N}}(y)\right) & =2\left(b_{\gamma+1}, \ldots, b_{N}\right)
\end{aligned}
$$

or equivalently

$$
\sum_{\beta=1}^{N} y_{\beta}\left(\left\|\left[\hat{Q}_{\alpha}, \hat{Q}_{\beta}\right]\right\|^{2}\right)-1= \begin{cases}a & \text { if } \alpha \leq \gamma \\ b_{\alpha} & \text { if } \alpha>\gamma\end{cases}
$$

Hence

$$
f_{Q}(y)=\left(\sum_{\alpha=1}^{\gamma} y_{\alpha}\right) a+\left(\sum_{\alpha=\gamma+1}^{N} b_{\alpha}\right) \varepsilon=0 \quad \text { and } \quad \sum_{\alpha=1}^{\gamma} y_{\alpha}+(N-\gamma) \varepsilon=1 .
$$

Meanwhile, we see $\partial f_{Q} / \partial v(y)=2\left(a \gamma+\sum_{\alpha=\gamma+1}^{N} b_{\alpha}\right) \leq 0$, where $v=(1, \ldots, 1)$ is the vector normal to $\triangle$ in $\mathbb{R}^{N}$. For any sufficiently small $\varepsilon$ (such as $\varepsilon<1 / N$ ), it follows from the above three formulas that $a \geq 0$. Without loss of generality, we assume $y_{1}=\max \left\{y_{1}, \ldots, y_{\gamma}\right\}>\varepsilon$. Let $J:=\left\{\beta \in S \mid\left\|\left[\hat{Q}_{1}, \hat{Q}_{\beta}\right]\right\|^{2} \geq 1\right\}$, and let 
$n_{1}$ be the number of elements of $J$. Now combining Lemma 2.3, Lemma 2.4 and Equation (3-5) will give a contradiction as follows:

$$
\begin{aligned}
& 1 \leq 1+a=\sum_{\beta=2}^{N} y_{\beta}\left\|\left[\hat{Q}_{1}, \hat{Q}_{\beta}\right]\right\|^{2} \\
&=\sum_{\beta \in J} y_{\beta}\left(\left\|\left[\hat{Q}_{1}, \hat{Q}_{\beta}\right]\right\|^{2}-1\right)+\sum_{\beta \in J} y_{\beta}+\sum_{\beta \in S / J} y_{\beta}\left\|\left[\hat{Q}_{1}, \hat{Q}_{\beta}\right]\right\|^{2} \\
& \leq y_{1} \sum_{\beta \in J}\left(\left\|\left[\hat{Q}_{1}, \hat{Q}_{\beta}\right]\right\|^{2}-1\right)+\sum_{\beta \in J} y_{\beta}+\sum_{\beta \in S / J} y_{\beta}\left\|\left[\hat{Q}_{1}, \hat{Q}_{\beta}\right]\right\|^{2} \\
&(3-6) \quad \leq y_{1}+\sum_{\beta \in J} y_{\beta}+\sum_{\beta \in S / J} y_{\beta}\left\|\left[\hat{Q}_{1}, \hat{Q}_{\beta}\right]\right\|^{2} \leq \sum_{\beta=1}^{N} y_{\beta}=1 .
\end{aligned}
$$

Thus

$$
y_{\beta}=y_{1} \quad \text { for } \beta \in J \quad \text { and } \quad \sum_{\beta \in J}\left\|\left[\hat{Q}_{1}, \hat{Q}_{\beta}\right]\right\|^{2}=n_{1}+1 \leq n<N .
$$

Hence $S /(J \cup\{1\}) \neq \varnothing$, and the second " $\leq$ " in line (3-6) should be " $<$ " by the definition of $J$ and the positivity of $y_{\beta}$ for $\beta \in S /(J \cup\{1\})$.

Now we consider the equality condition of Conjecture A in view of the proof of the a priori estimate.

If there's an orthogonal matrix $Q$ and a point $y \in \Delta$ such that $f_{Q}(y)=0$, we can assume without loss of generality that

$$
y \in \Delta^{\gamma}:=\left\{x \in \Delta \mid x_{\alpha}>0 \text { for all } \alpha \leq \gamma \text { and } x_{\beta}=0 \text { for all } \beta>\gamma\right\}
$$

for some $1 \leq \gamma \leq N$. Then $y$ is a maximum point of $f_{Q}(x)$ in $\mathbb{R}_{+}^{N}$ and an interior maximum point in $\Delta^{\gamma}$. Therefore, we have the same conclusions as (3-4), (3-5), (3-6), and (3-7) when $\gamma=n_{1}+1$, and all inequalities in the proof of Lemma 2.3 can be replaced by equalities. So $\sum_{\beta \in J} q_{\gamma \beta}^{2}=1$ implies $q_{\gamma \beta}=0$ for all $\gamma \in I$ and $\beta \in S / J$. And also it follows from Lemma 2.2 that $\left(\lambda_{i}-\lambda_{j}\right)^{2}-1<0$ for all $\gamma=(i, j) \in S / I$, and thus $q_{\gamma \beta}=0$ for all $\beta \in J$ and $\gamma \in S / I$. Hence $n_{0}=n_{1}$, and it follows from Lemma 2.1 that all $\hat{Q}_{\beta}$ for $\beta \in J$ have rank 2. On the other hand, we know from the first formula of (3-7) that $\hat{Q}_{1}$ can be replaced in the above arguments by $\hat{Q}_{\beta}$ for some $\beta \in J$, which implies that $\hat{Q}_{1}$ has rank 2 and thus $n_{0}=n_{1}=1$ and $\gamma=2$. Finally we can conclude the equality case of Theorem 1.1 from Lemmas 2.2 and 2.6.

Proof of Corollary 1.2. The inequality case is equivalent to that of Theorem 1.1; see also [Dillen et al. 2007, Theorem 3.1]. When the equality holds at some point $p \in M$, we can choose an orthonormal basis $\left\{u_{1}, \ldots, u_{m}\right\}$ of $T_{p}^{\perp} M$ such that $u_{1}=H /|H|$ if $H \neq 0$ or arbitrarily if $H=0$. Put $B_{1}=A_{u_{1}}-|H| I_{n}$ and $B_{r}=A_{u_{r}}$ for $2 \leq r \leq m$. Applying the equality case of Theorem 1.1 and choosing an orthonormal basis $\left\{e_{1}, \ldots, e_{n}\right\}$ of $T_{p} M$, we get $\left(B_{1}, \ldots, B_{m}\right)=\left(H_{1}, H_{2}, 0, \ldots, 0\right) R$ for some $m \times m$ orthogonal matrix $R=\left(R_{r s}\right)$. Therefore $\left(A_{u_{1}}, \ldots, A_{u_{m}}\right) R^{t}=$ $\left(H_{1}+|H| R_{11} I_{n}, H_{2}+|H| R_{21} I_{n},|H| R_{31} I_{n}, \ldots,|H| R_{m 1} I_{n}\right)$. Then taking the orthonormal basis $\left(\xi_{1}, \ldots, \xi_{m}\right)=\left(u_{1}, \ldots, u_{m}\right) R^{t}$ of $T_{p}^{\perp} M$ completes the proof. 


\section{Acknowledgments}

We thank Professor Weiping Zhang for his encouragement and for introducing us to [Lu 2007c]. Many thanks as well to Professors Marcos Dajczer and Ruy Tojeiro and the referee for their useful comments and suggestions on the previous version of this paper.

\section{References}

[Chern 1968] S. S. Chern, Minimal submanifolds in a Riemannian manifold, University of Kansas Lawrence, 1968. MR $40 \# 1899$

[Choi and Lu 2008] T. Choi and Z. Lu, "On the DDVV conjecture and the comass in calibrated geometry, I”, Math. Z. (2008). arXiv math/0610709

[Dajczer and Florit 2001] M. Dajczer and L. A. Florit, "A class of austere submanifolds", Illinois J. Math. 45:3 (2001), 735-755. MR 2003g:53090 Zbl 0988.53004

[DDVV 1999] P. J. De Smet, F. Dillen, L. Verstraelen, and L. Vrancken, "A pointwise inequality in submanifold theory”, Arch. Math. (Brno) 35:2 (1999), 115-128. MR 2000h:53072 Zbl 1054.53075

[Dillen et al. 2007] F. Dillen, J. Fastenakels, and J. Veken, "Remarks on an inequality involving the normal scalar curvature", pp. 83-92 in Proceedings of the International Congress on Pure and Applied Differential Geometry PADGE (Brussels, 2007), edited by F. Dillen and I. Van de Woestyne, Shaker, Aachen, 2007. Zbl 05244758 arXiv math/0610721v2

[Lu 2007a] Z. Lu, "On the DDVV conjecture and the comass in calibrated geometry, II", Preprint, 2007. arXiv 0708.2921v1

[Lu 2007b] Z. Lu, "Proof of the normal scalar curvature conjecture", Preprint, 2007. arXiv 0711. 3510v1

[Lu 2007c] Z. Lu, "Recent developments of the DDVV conjecture”, Preprint, 2007. arXiv 0708. 3201v1

Received November 21, 2007. Revised February 12, 2008.

GE JIANQUAN

DEPARTMENT OF MATHEMATICAL SCIENCES

TSINGHUA UNIVERSITY

BEIJING 100084

CHINA

gejq04@mails.tsinghua.edu.cn

TANG ZIZHOU

School of Mathematical Sciences

LABORATORY OF MATHEMATICS AND COMPLEX SYSTEMS

BEIJING NORMAL UNIVERSITY

BEIJING 100875

CHINA

zztang@mx.cei.gov.cn 